\title{
MASTIGAÇÃO E ATIVIDADE ELETROMIOGRÁFICA EM CRIANÇAS COM MORDIDA CRUZADA POSTERIOR
}

\author{
Mastication and electromyographic activity \\ in children with posterior crossbite
}

\author{
Luciana Vitaliano Voi Trawitzki (1), Cláudia Maria de Felício (2), \\ Regina Maria Puppin-Rontani ${ }^{(3)}$, Mirian Aiko Nakane Matsumoto ${ }^{(4)}$, Mathias Vitti ${ }^{(5)}$
}

\section{RESUMO}

Objetivo: investigar a preferência mastigatória e o comportamento dos músculos mastigatórios, em crianças de 6 a 9 anos, com mordida cruzada posterior. Métodos: 30 crianças foram selecionadas num serviço de Ortodontia de uma universidade pública. Após a concordância na participação no trabalho, foi realizada entrevista com a criança e seu responsável, para investigação de disfunção temporomandibular; análise da preferência mastigatória, por meio de registros em vídeo e avaliação eletromiográfica (EMG) dos músculos masseter e temporal anterior, durante a mastigação solicitada, direita e esquerda, de uma goma de marcar. Resultados: houve diferença significante na atividade EMG dos músculos masseter e temporal anterior entre os lados de trabalho e balanceio, porém não houve diferença estatística quando foram comparadas as atividades EMG entre os lados de mordida cruzada e não cruzada, tampouco entre os lados de preferência e não preferência mastigatória. Conclusão: na amostra estudada não se verificou assimetria funcional muscular estabelecida.

DESCRITORES: Eletromiografia; Músculos; Mastigação

(1) Fonoaudióloga; Professora Doutora do Departamento de Oftalmologia, Otorrinolaringologia e Cirurgia de Cabeça e Pescoço da Faculdade de Medicina de Ribeirão Preto da Universidade de São Paulo, FMRP-USP, Ribeirão Preto, SP; Doutora em Biociências Aplicada à Clínica Médica pela Faculdade de Medicina de Ribeirão Preto da Universidade de São Paulo.

(2) Fonoaudióloga; Professora Doutora do Departamento de Oftalmologia, Otorrinolaringologia e Cirurgia de Cabeça e Pescoço da Faculdade de Medicina de Ribeirão Preto da Universidade de São Paulo, FMRP-USP, Ribeirão Preto, SP; Doutora em Ciências (Psicobiologia) pela Faculdade de Filosofia Ciências e Letras de Ribeirão Preto da Universidade de São Paulo.

(3) Cirurgiã Dentista; Professora Livre Docente do Departamento Odontologia Infantil da Faculdade de Odontologia de Piracicaba da Universidade Estadual de Campinas, FOP-UNICAMP, Piracicaba, SP.

(4) Cirurgiã Dentista; Professora Livre Docente do Departamento de Clínica Infantil e Odontologia Preventiva e Social da Faculdade de Odontologia de Ribeirão Preto da Universidade de São Paulo, FORP-USP, Ribeirão Preto, SP.

(5) Cirurgião Dentista; Professor Titular do Departamento de Morfologia, Estomatologia e Fisiologia da Faculdade de Odontologia de Ribeirão Preto da Universidade de São Paulo, FORP-USP, Ribeirão Preto, SP.

Conflito de interesses: inexistente

\section{INTRODUÇÃO}

O crescimento craniofacial é um processo que visa alcançar o equilíbrio funcional e estrutural. A resposta esquelética craniofacial é resultado das ações de crescimento e contínuo remodelamento, reguladas pelas funções orofaciais e pelo crescimento dos músculos da mastigação, das vias aéreas, mucosas, músculos faríngeos, tecidos conjuntivos, entre outros ${ }^{1}$.

Alterações na oclusão dentária e na morfologia facial podem ser decorrentes de problemas respiratórios causados por obstrução nasal ${ }^{2,3}$, e ainda por influências de hábitos orais deletérios como os de sucção ${ }^{3-11}$, assim como de padrões alterados de deglutição ${ }^{7}$ e de mastigação ${ }^{12}$.

A função mastigatória pode ser também influenciada por diversos fatores, sendo um deles a oclusão dentária ${ }^{12-14}$.

Embora a performance mastigatória melhore com a idade, pois está relacionada com o tamanho corpóreo, sendo independentemente do gênero ${ }^{15}$, 
a má oclusão pode afetar negativamente a habilidade no processo de quebra dos alimentos ${ }^{13}$.

Pesquisadores investigaram a relação da função mastigatória com a morfologia orofacial em crianças em fase de crescimento ${ }^{16-22} \mathrm{e}$ em adultos jovens ${ }^{14,23-27}$.

O comportamento miofuncional orofacial por meio de exames objetivos tem sido objeto de estudo dos pesquisadores, em crianças com mordida cruzada posterior, justificado por sua prevalência na população urbana.

Algumas pesquisas feitas em crianças com mordida cruzada posterior reportaram a presença de deslocamentos mandibulares durante a protrusão e fechamento da mandíbula, com padrão anormal de deglutição ${ }^{16}$; velocidade mais lenta nos ciclos mastigatórios e maior excursão dos movimentos mandibulares ${ }^{18}$; valores menores de máxima força de mordida ${ }^{28}$ e valores maiores de atividade EMG do masseter e digástrico do lado direito durante a mastigação ${ }^{29}$.

Em adultos jovens, Ferrario et al ${ }^{23}$ verificaram que a mordida cruzada posterior unilateral afeta a coordenação muscular e segundo Miyawaki et al ${ }^{24}$, interfere no deslocamento do côndilo mandibular durante a mastigação, assim como na morfologia do ciclo mastigatório ${ }^{14}$. Em contrapartida, algumas pesquisas não encontraram diferenças em crianças com mordida cruzada posterior comparativamente a um grupo controle em relação ao lado de preferência mastigatória ${ }^{16,29}$, na extensão dos movimentos mandibulares ${ }^{16,19}$, na atividade EMG dos músculos temporal e masseter na posição de repouso, atividade EMG do temporal durante a mastigação e do masseter durante a deglutição, nem entre os lados direito e esquerdo para ambos os músculos, durante a mastigação ${ }^{29}$. O estudo de Sonnesen et al ${ }^{28}$ também não encontrou diferença significativa na força máxima de mordida entre o lado de mordida cruzada e o lado não cruzado.

A influência do tratamento ortodôntico na função mastigatória em crianças com mordida cruzada posterior ainda é pouco conhecida. Num estudo de caso, Saitoh et al ${ }^{30}$ não encontraram melhora total no padrão mastigatório. Entretanto, Throckmorton et al ${ }^{21}$ revelaram uma melhora na duração e na forma dos ciclos mastigatórios e Piancino et al ${ }^{12}$ demonstraram diferença na morfologia do ciclo mastigatório antes e após a terapia ortodôntica, apenas no lado cruzado. O tratamento precoce da mordida cruzada posterior deve ser preconizado ${ }^{28,30}$ evitando-se alterações musculares e funcionais na fase adulta ${ }^{28}$.

Diante do exposto e visando uma melhor compreensão da função mastigatória em crianças com mordida cruzada posterior, o presente estudo buscou investigar a preferência mastigatória e a atividade EMG dos músculos masseter e temporal anterior, durante a mastigação, e suas relações com a mordida cruzada posterior.

\section{MÉTODOS}

Participaram do estudo 30 crianças portadoras de mordida cruzada posterior, sendo 19 do gênero feminino e 11 do gênero masculino, na faixa etária de 6 anos a 9 anos e 3 meses (média de 8 anos e 1 mês), as quais aguardavam o tratamento ortodôntico na Clínica de Ortodontia Preventiva da Faculdade de Odontologia de Ribeirão Preto da Universidade de São Paulo (FORP-USP). Para a seleção das crianças, foram analisados previamente os modelos de gesso de pacientes que em máxima intercuspidação apresentaram mordida cruzada posterior (uni ou bilateral), associado ou não a outro tipo de má oclusão, sem ausência dentária posterior.

Adotou-se como critérios de exclusão a presença de sinais e sintomas de disfunção temporomandibular (DTM), síndromes genéticas, deformidades craniofaciais como fissuras labiopalatinas, cáries dentárias, distúrbios neuromusculares, distúrbios cognitivos e/ ou mentais, e crianças que já haviam realizado qualquer tratamento fonoaudiológico ou ortodôntico.

Após a seleção das crianças e concordância das mesmas e de seus responsáveis, aplicou-se um questionário, contendo questões sobre aspectos neuromusculares referentes à dor ou fadiga na região dos músculos masseter e temporal anterior, investigação de sinais e sintomas de DTM, como presença de dor ou desconforto, presença de ruídos articulares e se a criança havia sido submetida a tratamento ortodôntico e/ou fonoaudiológico.

Realizou-se a avaliação do tipo mastigatório, por meio da solicitação da mastigação habitual, fornecendo a cada criança um tablete da goma de mascar Trident (Warner-Lambert, Divisão Adams, Bauru, São Paulo, Brasil) sabor menta. As crianças permaneceram sentadas e a mastigação foi registrada com uma câmara de vídeo (handcanzoom 10x-SONY) instalada à sua frente, sobre um tripé. A imagem foi analisada posteriormente. Para interpretação dos dados foi considerado o lado de preferência mastigatória aquele em que ocorreu no mínimo $66 \%$ do total de golpes mastigatórios, como considerado por Felício ${ }^{31}$. Uma porcentagem mais equilibrada entre os lados, ou se ambos os lados eram utilizados simultaneamente, foi considerado mastigação bilateral.

No Laboratório de Eletromiografia e Diagnóstico Oclusal Computadorizado, do Departamento de Morfologia, Estomatologia e Fisiologia da 
FORP-USP, registrou-se os potenciais elétricos musculares, por meio da EMG. Utilizou-se o eletromiógrafo modelo K6-I Light Channel Surface Eletromyography (Myo-Tronics Co. Sattle, WA, EUA), de oito canais, com scanner \#9, calibrado a uma amplitude de 500 microvolts.

Para captação dos sinais elétricos dos músculos masseter $(\mathrm{M})$ e temporal - feixe anterior $(\mathrm{T})$, direito (D) e esquerdo (E), foram empregados eletrodos duplos de superfície de Cloreto de Prata descartáveis (Duo-Trodes, Myo-Tronics, Seattle, WA) contendo um gel condutor (Myo-gel, Myo-Tronics, Seattle,WA) e um eletrodo terra foi usado na região do pescoço do lado esquerdo. Os eletrodos foram fixados e posicionados em paralelo às fibras musculares de acordo com a recomendação do fabricante. Os sítios de superfície da pele foram limpos previamente com algodão embebido em álcool 70 GL, para remoção dos resíduos de gordura presentes na pele.

Proporcionou-se um ambiente calmo e descontraído e as crianças foram orientadas a permanecerem sentadas em uma cadeira tipo escritório confortável, em postura ereta com as mãos apoiadas sobre as coxas e com a planta dos pés apoiadas no solo. A postura de cabeça também ereta, com o plano de Camper paralelo ao solo. A examinadora monitorou a postura das crianças durante toda a avaliação, objetivando minimizar a interferência de estímulos externos.

Os registros de atividade EMG foram obtidos durante a mastigação da goma de mascar. Deuse a goma para a criança e solicitou-se que mastigasse por um tempo médio de 15 segundos, para obter uma consistência uniforme, antes dos registros. As crianças foram orientadas para a realização do teste, individualmente, solicitando que mastigassem com os dentes posteriores, iniciando pelo lado direito, até que fossem interrompidas e depois fizessem o mesmo do lado esquerdo.

O presente estudo foi aprovado pelo Comitê de Ética em Pesquisa da Faculdade de Odontologia de Piracicaba da Universidade de Campinas (FOPUNICAMP), sob o protocolo no 22/99. Realizou-se o esclarecimento da pesquisa ao responsável e à criança, e assim foram convidados a participar voluntariamente. Os responsáveis assinaram o termo de Consentimento Livre e Esclarecido consentindo a participação na pesquisa e a divulgação dos resultados, conforme Resolução 196/96.

O teste estatístico de Wilcoxon foi utilizado na comparação dos valores de atividade $\mathrm{EMG}, \mathrm{em} \mu \mathrm{V}$, dos músculos $\mathrm{M}$ e $\mathrm{T}$, entre os lados de trabalho e balanceio para todas as crianças da amostra, entre os lados de mordida cruzada e não cruzada para as crianças com mordida cruzada posterior unilateral e entre os lados de preferência e não preferência mastigatória para as crianças que apresentaram preferência mastigatória unilateral. Também foi utilizado o teste Exato de Fisher para verificar diferença estatística entre os grupos com mordida cruzada posterior quanto à preferência mastigatória. Usouse o programa estatístico GMC Basic Sotware versão 7.3 .

\section{RESULTADOS}

Das 30 crianças selecionadas com mordida cruzada posterior, $17(56,66 \%)$ apresentaram mordida cruzada posterior direita (MCPD), oito crianças $(26,66 \%)$ mordida cruzada posterior esquerda (MCPE) e cinco crianças (16,66\%) mordida cruzada posterior bilateral (MCPB). Associadas a mordida cruzada posterior, duas crianças $(6,66 \%)$ apresentavam mordida cruzada anterior e seis crianças $(20 \%)$ apresentavam mordida aberta anterior.

A atividade EMG no lado de trabalho foi significativamente maior $(p<0,01)$ em comparação ao lado de balanceio para os músculos M e T. Na Tabela 1 encontram-se os resultados.

Considerando-se as 25 crianças com mordida cruzada unilateral, comparou-se a atividade EMG dos músculos $\mathrm{M}$ e T, entre os lados cruzado e não cruzado, na situação de trabalho e balanceio e não houve diferença estatisticamente significante $(p>0,05)$ (Tabela 2).

Tabela 1 - Valores medianos da amplitude da atividade EMG, em $\mu \mathrm{V}$, do músculo masseter e temporal anterior obtidos para os lados de trabalho e balanceio

\begin{tabular}{lccc}
\hline & Trabalho & Balanceio & p \\
\hline Masseter & 30,7 & 14,8 & $\mathrm{p}<0,01^{*}$ \\
$\begin{array}{l}\text { Temporal Anterior } \\
\mathrm{n}=30\end{array}$ & 25,5 & 16,6 & $\mathrm{p}<0,01^{*}$ \\
\hline
\end{tabular}

*Diferença estatisticamente significante

Teste de Wilcoxon

Rev CEFAC, v.11, Supl3, 334-340, 2009 
Tabela 2 - Valores medianos da amplitude da atividade EMG, em $\mu \mathrm{V}$, do músculo masseter e temporal anterior, obtidos para os lados cruzado e não cruzado, na situação de trabalho e balanceio

\begin{tabular}{lccc}
\hline \multicolumn{1}{c}{ Lado cruzado } & Lado não cruzado & $\mathbf{p}$ \\
\hline \multicolumn{1}{c}{ Masseter } & & \\
Trabalho & 35,0 & 32,8 & $\mathrm{p}>0,05$ \\
Balanceio & 11,2 & 15,6 & $\mathrm{p}>0,05$ \\
$\quad$ Temporal Anterior & & & $\mathrm{p}>0,05$ \\
$\begin{array}{l}\text { Trabalho } \\
\text { Balanceio }\end{array}$ & 22,6 & 25,0 & $\mathrm{p}>0,05$ \\
$\mathrm{n}=25$ & 16,5 & 15,5 & \\
\hline
\end{tabular}

Teste de Wilcoxon

Das 30 crianças analisadas, observou-se que sete delas $(23,4 \%)$ apresentaram a preferência mastigatória pelo lado direito (PMD), sete $(23,4 \%)$ preferência pelo lado esquerdo (PME) e 16 (53,4\%) preferência mastigatória bilateral (PMB).

Considerando-se apenas as 14 crianças com preferência mastigatória unilateral comparou-se a atividade EMG dos músculos $\mathrm{M}$ e $\mathrm{T}$, durante a mastigação, entre os lados de preferência e não preferência mastigatória. Não houve diferença estatisticamente significante $(p>0,05)$ entre os referidos lados, para ambos os músculos. Os resultados encontram-se na Tabela 3.
$\mathrm{Na}$ Tabela 4 pode-se observar a distribuição da amostra de acordo com a localização da mordida cruzada e a mastigação. Não houve diferença estatística significante $(p>0,05)$ entre os grupos com mordida cruzada unilateral direita e esquerda quanto ao lado de preferência mastigatória, de acordo com o teste Exato de Fisher. Assim, eles foram agrupados em um único grupo de mordida cruzada unilateral o qual foi comparado ao grupo com mordida cruzada bilateral, não havendo diferença estatística entre eles quanto ao lado de preferência mastigatória (direito ou esquerdo), nem quanto à mastigação unilateral e bilateral $(p>0,05)$.

Tabela 3 - Valores medianos da amplitude da atividade EMG, em $\mu \mathrm{V}$, do músculo masseter e temporal anterior obtidos para os lados de preferência e não preferência mastigatória

\begin{tabular}{lccc}
\hline & Lado preferência & Lado não preferência & $\mathbf{p}$ \\
\hline Masseter & 28,5 & 37,8 & $\mathrm{p}>0,05$ \\
Temporal Anterior & 26,6 & 24,4 & $\mathrm{p}>0,05$ \\
$\mathrm{n}=14$ & & & \\
\hline
\end{tabular}

Teste de Wilcoxon

Tabela 4 - Distribuição da amostra de acordo com a localização da mordida cruzada e a preferência mastigatória

\begin{tabular}{lccc}
\hline & PMD & PME & PMB \\
\hline MCPD $(n=17)$ & 3 & 5 & 9 \\
MCPE $(n=8)$ & 2 & 1 & 5 \\
MCPB $(n=5)$ & 2 & 1 & 2 \\
\hline
\end{tabular}

MCP: mordida cruzada posterior; PM: preferência mastigatória; D: direita; E: esquerda; B: bilateral.

\section{DISCUSSÃO}

No presente estudo, verificou-se que a atividade EMG do lado de trabalho foi significativamente maior que do lado de balanceio, tanto para o músculo $\mathrm{M}$ quanto para o músculo $\mathrm{T}$. Observa-se que apesar da morfologia oclusal alterada, na amostra estudada, manteve-se um padrão de atividade 
muscular sincrônico. Nos estudos de Ferrario et al ${ }^{23} \mathrm{e}$ Trawitzki et al ${ }^{26}$ que analisaram a atividade EMG dos músculos $\mathrm{M}$ e T, entre os lados, durante a mastigação de goma, em adultos jovens com alterações na morfologia oclusal, foram verificadas irregularidades na contração desses músculos entre os lados. A morfologia dentofacial alterada na fase adulta evidencia uma contração muscular assincrônica e ainda pode-se atribuir a grande variabilidade nos resultados musculares, às diferenças biológicas individuais ${ }^{23}$. O mesmo parece não ser evidente em crianças, na faixa etária estudada.

Alarcón et al ${ }^{29}$ não encontraram diferenças estatisticamente significantes na atividade EMG dos músculos $\mathrm{T}$ (feixes anterior e posterior) e $\mathrm{M}$ entre os lados, durante a mastigação, demonstrando uma atividade muscular simétrica tanto em crianças com mordida cruzada quanto em indivíduos controles. Sonnesen et al ${ }^{28}$ também não demonstraram diferenças significantes na força máxima de mordida entre os lados, em crianças de 7 a 13 anos, com mordida cruzada posterior unilateral.

O estudo de Rilo et al ${ }^{14}$ investigou parâmetros objetivos da função mastigatória, por meio da morfologia do ciclo e distância do contato da guia oclusal durante o fechamento mandibular em adultos jovens com mordida cruzada. Participaram da pesquisa 25 pacientes, de 17 a 26 anos, com um ou mais dente cruzado, na região posterior. Os autores identificaram associação entre a morfologia do ciclo mastigatório e o número de dentes envolvendo a mordida cruzada, ou seja, quanto mais dentes cruzados, maior a proporção de ciclos mastigatórios anormais.

Participaram do estudo, crianças com mordida cruzada posterior independente do número de dentes cruzados e de alterações morfológicas no sentido vertical de crescimento da face, como dólico, meso e braquifacial. $\mathrm{O}$ estudo de Trawitzki et al ${ }^{17}$ em crianças com mordida cruzada posterior observou um predomínio de crianças com tendências de crescimento dolicofacial e uma minoria com tendência braquifacial. Os autores justificaram a relação da respiração oral prolongada na infância, com alterações no esqueleto da face, associando os padrões alterados nos sentidos transversal e vertical.

Como a presença de mordida cruzada, uni ou bilateral, poderia influenciar no padrão de mastigação, no presente estudo procurou-se adequar e subdividir os grupos em cada análise, ou seja, para verificar diferença na atividade EMG entre lados cruzado e não cruzado foram mantidas para análise apenas as crianças com mordida cruzada unilateral e foram excluídas para essa análise cinco crianças, que tinham mordida cruzada bilateral.
Subjacente ao objetivo de verificar se a atividade EMG durante a mastigação do lado cruzado diferia da atividade do lado não cruzado, ambos como lado de trabalho, estava a necessidade de compreender se crianças com esse tipo de má oclusão apresentavam alguma assimetria na atividade muscular, mas esta não foi detectada entre os indivíduos com mordida cruzada unilateral, na faixa etária estudada.

Os achados do presente estudo corroboram o estudo de Alarcón et al ${ }^{29}$ que também não identificou diferenças significantes na atividade EMG dos músculos temporal e masseter em crianças com mordida cruzada posterior entre os lados, cruzado e não cruzado, durante a mastigação. Os autores identificaram apenas maiores valores do lado não cruzado para o músculo temporal posterior, durante o repouso e deglutição. Sonnesen et al ${ }^{28}$ também não encontraram diferenças estatisticamente significantes na força máxima de mordida entre os lados de mordida cruzada e não cruzada.

Ferrario et al ${ }^{23}$ verificaram que em adultos jovens a mordida cruzada pode influenciar a função muscular. Esses resultados divergem dos encontrados no presente estudo, o que parece estar relacionado à fase de desenvolvimento do sistema estomatognático, visto que a amostra estudada apresentava média de idade de 8 anos e 1 mês, isto é, antes do surto de crescimento da puberdade.

A não diferenciação entre a atividade EMG do lado cruzado e não cruzado talvez possa também ser explicada pelo fato de que, dos 25 indivíduos com mordida cruzada unilateral, apenas 11 apresentavam preferência mastigatória unilateral, sendo que desses, sete indivíduos apresentaram preferência contralateral à mordida cruzada. Assim, supõe-se que se vários indivíduos exercitavam a musculatura mastigatória bilateralmente, ou ainda contralateral à mordida cruzada, a probabilidade de haver diferença na atividade EMG entre os lados, cruzado e não cruzado tornou-se reduzida.

Também, quando considerado para a análise apenas os 14 indivíduos com preferência mastigatória unilateral, não foi observada diferença significativa na atividade EMG dos músculos $M$ entre os lados de preferência e não preferência mastigatória, tampouco dos músculos $T$, confirmando não haver assimetria na função muscular, nessa amostra. Nenhum estudo foi encontrado com a metodologia usada, investigando a atividade EMG entre os lados de preferência e não preferência mastigatória. Estudos semelhantes indicaram um padrão de mastigação bilateral em crianças com mordida cruzada posterior não diferindo de indivíduos controles ${ }^{16,29}$.

No presente estudo não houve associação entre o lado de mordida cruzada e a preferência mastigatória. $O$ estudo de Martin et al ${ }^{16}$ também não 
encontrou relação entre o lado de mordida cruzada e a preferência mastigatória, em crianças de 10 a 14 anos de idade.

Tendo em vista os dados da literatura de que existe, em adultos, assimetria funcional nos casos de mordida cruzada ${ }^{23}$ e diferenças entre os indivíduos dólico, meso e braquifaciais, quanto à capacidade do músculo para produzir atividade, mas não em crianças ${ }^{17}$, supõe-se que uma vez instalada a má oclusão, a musculatura tenderá progressivamente a se adaptar, devido às compensações necessárias para as realizações das funções estomatognáticas, dentre elas a mastigação, manifestando-se o problema muscular numa fase posterior à infância. Embora se sabe, com base na literatura, que a mastigação de alimentos consistentes contribui para o crescimento e desenvolvimento craniofacial, a partir dos resultados obtidos, sugere-se que em casos de mordida cruzada posterior o estresse mastigatório seja evitado, antes da correção ortodôntica, pois a maior demanda poderia levar mais rapidamente a uma adaptação, e consequentemente, à assimetria miofuncional.

\section{CONCLUSÃO}

A amostra estudada, de crianças com mordida cruzada posterior, não apresentou assimetria funcional muscular estabelecida, visto que não houve diferença estatística significante quando se comparou a atividade EMG dos músculos $\mathrm{M}$ e $\mathrm{T}$ entre os lados de mordida cruzada e não cruzada, lados de preferência e não preferência mastigatória. Também não houve associação entre a mordida cruzada e a preferência mastigatória.

\begin{abstract}
Purpose: to investigate the masticatory preference and the behavior of masticatory muscles, in children between 6 to 9 -year old, with posterior crossbite. Methods: 30 children were selected from the Orthodontical service of a public university. After consenting to take part in the study, there was an interview with the children and the parent, in order to investigate temporomandibular disorders; masticatory was analyzed through video recording and electromyographic (EMG) evaluation of the masseter and anterior temporal, during the solicited mastication, on right and left, using chewing gum. Results: there was a significant difference in the EMG activity of the masseter and temporal between work and balance sides, however there was no statistical differences in the comparison between crossbite side and no crossbite side, but neither between preference side and non the preference side. Conclusion: in the studied population there was no functional muscular asymmetry established.
\end{abstract}

KEYWORDS: Electromyography; Muscles; Mastication

\section{REFERÊNCIAS}

1. Enlow DH, Hans MG. Noções básicas sobre crescimento facial. São Paulo: Santos Livraria Editora; 1998. 304p.

2. Lessa FCR, Enoki C, Feres MFN, Valera FCP, Anselmo-Lima WT, Matsumoto MAN. Influência do padrão respiratório na morfologia craniofacial. Rev Bras Otorrinolaringol. 2005; 71(2):156-60.

3. Vázquez-Nava F, Quezada-Castillo JA, OviedoTreviño S, Saldivar-González AH, Sánchez-Nuncio HR, Beltrán-Guzmán FJ, et al. Association between allergic rhinitis, bottle feeding, non-nutritive sucking habits, and malocclusion in the primary dentition. Arch Dis Child. 2006; 91(10):836-40.

4. Degan VV, Puppin-Rontani RM. Prevalence of pacifier-sucking habits and successful methods to eliminate them - a preliminary study. J Dent Child. 2004; 71(2):148-51.

5. Caglar E, Larsson E, Andersson EM, Hauge MS, Ogaard B, Bishara S, et al. Feeding, artificial sucking habits, and malocclusions in 3-year-old girls in different regions of the world. J Dent Child. 2005; 72(1):25-30.

6. Cozza P, Baccetti T, Franchi L, Mucedero M, Polimeni A. Sucking habits and facial hyperdivergency as risk factors for anterior open bite in the mixed dentition. Am J Orthod Dentofacial Orthop. 2005; 128(4):517-9.

7. Degan VV, Puppin-Rontani RM. Remoção de hábitos e terapia miofuncional: restabelecimento da deglutição e repouso lingual. Pró-Fono. 2005; 17(3):375-82. 
8. Katz CR, Rosenblatt A. Nonnutritive sucking habits and anterior open bite in Brazilian children: a longitudinal study. Pediatr Dent. 2005; 27(5):369-73. 9. Maciel CTV, Leite ICG. Aspectos etiológicos da mordida aberta anterior e suas implicações nas funções orofaciais. Pró-Fono. 2005; 17(3):293-302. 10. Warren JJ, Slayton RL, Bishara SE, Levy SM, Yonezu T, Kanellis MJ. Effects of nonnutritive sucking habits on occlusal characteristics in the mixed dentition. Pediatr Dent. 2005; 27(6):445-50.

11. Aznar T, Galán AF, Marín I, Domínguez A. Dental arch diameters and relationships to oral habits. Angle Orthod. 2006; 76(3):441-5.

12. Piancino MG, Talpone F, Dalmasso P, Debernardi C, Lewin A, Bracco P. Reverse-sequencing chewing patterns before and after treatment of children with a unilateral posterior crossbite. Eur J Orthod. 2006; 28(5):480-4.

13. English JD, Buschang PH, Throckmorton GS. Does malocclusion affect masticatory performance? Angle Orthod. 2002; 72(1):21-7.

14. Rilo B, Silva JL, Mora MJ, Cadarso-Suárez C, Santana U. Unilateral posterior crossbite and mastication. Arch Oral Biol. 2007; 52(5):474-8.

15. Toro A, Buschang PH, Throckmorton G, Roldán S. Masticatory performance in children and adolescents with Class I and II malocclusions. Eur J Orthod. 2006; 28(2):112-9.

16. Martín C, Alarcón JA, Palma JC. Kinesiographic study of the mandible in young patients with unilateral posterior crossbite. Am J Orthod Dentofacial Orthop. 2000; 118(5):541-8.

17. Trawitzki LVV, Puppin-Rontani RM, Felício $\mathrm{CM}$, Vitti M, Matsumoto MAN. Investigação eletromiográfica dos músculos masseter e temporal durante a mastigação em crianças com diferentes tendências de crescimento facial. Rev Soc Bras Fonoaudiol. 2000; 1(7):54-8.

18. Throckmorton GS, Buschang $\mathrm{PH}$, Hayasaki $\mathrm{H}$, Pinto AS. Changes in the masticatory cycle following treatment of posterior unilateral crossbite in children. Am J Orthod Dentofacial Orthop. 2001; 120(5):521-9.

RECEBIDO EM: 05/02/2009

ACEITO EM: 18/09/2009

Endereço para correspondência:

Luciana Vitaliano Voi Trawitzki

Av. Bandeirantes, 3900

Ribeirão Preto - SP

CEP: $14048-900$

E-mail: luvoi@fmrp.usp.br
19. Salioni MA, Pellizoni SE, Guimarães AS, Juliano Y, Alonso LG. Functional unilateral posterior crossbite effects on mastication movements using axiography. Angle Orthod. 2005; 75(3):362-7.

20. Sonnesen L, Bakke M. Molar bite force in relation to occlusion, craniofacial dimensions, and head posture in pre-orthodontic children. Eur J Orthod. 2005; 27(1):58-63.

21. Learreta JA, Bono AE, Maffia G, Beas J. The identification of temporomandibular joint disease through the masticatory cycle. Int $\mathrm{J}$ Orthod Milwaukee. 2005; 16(1):11-5.

22. Rodrigues AM, Bérzin F, Siqueira VCV. Análise eletromiográfica dos músculos masseter e temporal na correção da mordida cruzada posterior. Rev Dental Press Ortodon Ortop Facial. 2006; 11(3):55-62.

23. Ferrario VF, Sforza C, Serrao G. The influence of crossbite on the coordinated electromyographic activity of human masticatory muscles during mastication. J Oral Rehabil. 1999; 26(7):575-81.

24. Miyawaki S, Tanimoto Y, Araki Y, Katayama A, Kuboki T, Yamamoto TT. Movement of the lateral and medial poles of the working condyle during mastication in patients with unilateral posterior crossbite. Am J Orthod Dentofacial Orthop. 2004; 126(5):549-54.

25. Rodrigues KA, Ferreira LP. Masseter muscles electromyography study of individuals with and without malocclusion during dental clenching. Electromyogr Clin Neurophysiol. 2004; 44(5):271-5. 26. Trawitzki LV, Dantas RO, Mello-Filho FV, Marques Júnior W. Effect of treatment of dentofacial deformities on the electromyographic activity of masticatory muscles. Int $\mathrm{J}$ Oral Maxillofac Surg. 2006; 35(2):170-3.

27. Trawitzki LV, Dantas RO, Mello-Filho FV, EliasJúnior J. Effect of treatment of dentofacial deformity on masseter muscle thickness. Arch Oral Biol. 2006; 51(12):1086-92.

28. Sonnesen L, Bakke M, Solow B. Bite force in pre-orthodontic children with unilateral crossbite. Eur J Orthod. 2001; 23(6):741-9.

29. Alarcón JA, Martín C, Palma JC. Effect of unilateral posterior crossbite on the electromyographic activity of human masticatory muscles. Am $\mathrm{J}$ Orthod Dentofacial Orthop. 2000; 118(3):328-34.

30. Saitoh I, Hayasaki H, Iwase Y, Nakata M. Improvement in jaw motion following treatment of unilateral crossbite in a child with primary dentition: a case report. Cranio. 2002; 20(2):129-34.

31. Felício CM. Desordem temporomandibular: avaliação e casos clínicos. In: Junqueira P, Dauden ANBC. Aspectos atuais em terapia fonoaudiológica. São Paulo: Pancast; 2002. p.33-64. 